\title{
Red Ginseng Oil Attenuates Oxidative Stress and Offers Protection against Ultraviolet-Induced Photo Toxicity
}

\author{
H. M. Arif Ullah $\left(\mathbb{D},{ }^{1}\right.$ Yuan Yee Lee ${ }^{D},{ }^{1}$ Minki Kim ${ }^{D},{ }^{1}$ Tae-Wan Kim $\left(\mathbb{D},{ }^{1}\right.$ Evelyn Saba $\left(\mathbb{D},{ }^{2}\right.$ \\ Yi-Seong Kwak $\left({ }^{\circ},{ }^{3}\right.$ Mansur Abdullah Sandhu $\left(\mathbb{C}^{2},{ }^{2}\right.$ and Man Hee Rhee ${ }^{1}{ }^{1}$ \\ ${ }^{1}$ Laboratory of Physiology and Cell Signaling, College of Veterinary Medicine, Kyungpook National University, \\ Daegu 41566, Republic of Korea \\ ${ }^{2}$ Department of Veterinary Biomedical Sciences, Faculty of Veterinary and Animal Sciences, Pir Mehr Ali Shah-Arid \\ Agriculture University, Rawalpindi, Pakistan \\ ${ }^{3}$ ReD Headquarters, Korea Ginseng Cooperation, Daejeon 34520, Republic of Korea
}

Correspondence should be addressed to Man Hee Rhee; rheemh@knu.ac.kr

Received 7 January 2021; Accepted 11 June 2021; Published 5 July 2021

Academic Editor: Daniele Vergara

Copyright $@ 2021$ H. M. Arif Ullah et al. This is an open access article distributed under the Creative Commons Attribution License, which permits unrestricted use, distribution, and reproduction in any medium, provided the original work is properly cited.

Ginseng (Panax ginseng Meyer) is a well-known herbal medicine that has been used for a long time in Korea to treat various diseases. This study investigated the in vitro and in vivo protective effects of red ginseng extract (RGE) and red ginseng oil (RGO). Liver injury was produced in BALB/c mice by $400 \mathrm{mg} / \mathrm{kg}$ of acetaminophen intraperitoneal injection. The antioxidant effects of RGE and RGO on the free radicals 2,2-diphenyl-1-picryl-hydrazyl-hydrate (DPPH) and 2,2'-azino-bis-3ethylbenzothiazoline-6-sulfonic acid (ABTS) were measured. In addition, the hepatoprotective activities of RGE and RGO on liver markers, including alanine aminotransferase (ALT), aspartate aminotransferase (AST), and oxidative stress markers, including superoxide dismutase (SOD), catalase (CAT) enzyme activity, and 8-hydroxy-2-deoxyguanosine (8-OHdG) in serum and histopathological analysis, were evaluated. The protective effect of RGO on UV-induced phototoxicity was also evaluated in $\mathrm{Balb} / \mathrm{c}$ 3T3 mouse fibroblast cell line. RGE and RGO effectively inhibited the radicals DPPH and ABTS compared with ascorbic acid and trolox, respectively. Moreover, RGE and RGO significantly decreased the liver enzyme (ALT and AST) levels, increased the antioxidant enzyme (SOD and CAT) levels, and decreased the DNA oxidation product (8-OHdG) content in mice serum. RGO also exhibited protective effect against UV irradiation compared with chlorpromazine hydrochloride, a known phototoxic drug, in Balb/c 3T3 cell line. RGE and RGO possess antioxidant and hepatoprotective properties in mice, and RGO exerts nonphototoxic activity in Balb/c 3T3 cells.

\section{Introduction}

Ginseng has been used as an herbal medicine and a functional food in Asia for more than 200 years due to its beneficial effects [1-3]. Approximately 12 species of ginseng have been recognized, among which Panax ginseng Meyer (Korean ginseng) is well-known and widely consumed. Red ginseng is the most widely used ginseng product in Asia, especially Korea, due to its various pharmacological activities, including antidiabetic, anticancer, antiplatelet, antioxidant, and antiobesity effects [4-7]. The major chemical compounds in red ginseng are the key sources for its medicinal activities [1]. Red ginseng is produced from fresh ginseng using a repeated process of steaming and followed by drying process $[3,8]$. Ginseng is commonly consumed in the various forms including tablets, capsules, candies, jellies, and various types of fermented products [1].

Ginsenosides are the bioactive constituents of red ginseng and having biological properties. Various ginsenosides have been discovered in red ginseng extract (RGE) and used widely due to their specific pharmacological effects [9]. Studies reported that the major ginsenoside profiles of the RGE are Rg1, Re Rf, Rh1, Rg2, Rb1, Rc, Rb2, Rb3, Rd, and Rg3 $[1,10]$. In addition, based on gas chromatography analysis, 
it has been shown that the major constituents of red ginseng oil (RGO) are unsaturated fatty acid (linoleic acid), saturated fatty acid (palmitic acid), phytosterins (alpha-tocopherol, Beta-sitosterol, gamma-sitosterol, Stigmasterol), and hydrocarbon (bicyclo) $[11,12]$. It has been reported that red ginseng essential oil has antioxidant and hepatoprotective effects in hydrogen peroxide- $\left(\mathrm{H}_{2} \mathrm{O}_{2}-\right)$ treated HepG2 cells and carbon tetrachloride- $\left(\mathrm{CCl}_{4^{-}}\right)$treated mice [8]. Moreover, a recent study demonstrated the antimelanogenic activities of Korean red ginseng oil (RGO) in a UV-B-induced hairless mouse [13].

Oxidative stress is the result of the production of reactive oxygen species (ROS) and reactive nitrogen species generated by the normal metabolic process and by exogenous stimuli in the body. These free radicals can damage the structure and function of cells, and increased concentrations of free radicals in metabolism are responsible for the development of various diseases $[14,15]$. Antioxidant enzymes and antioxidant molecules can prevent or reduce the generation of these free radicals through the antioxidative defense system, which neutralizes ROS or scavenges free radicals $[14,16]$. Several previous studies have demonstrated that an overdose of acetaminophen (APAP) can increase ROS production, and as a result, the oxidative stress causes severe liver injury [1719]. APAP induces oxidative stress that can attack cell organelles, damage the cell membrane, induce lipid peroxidation, and ultimately result in liver injury [20]. Furthermore, high-energy visible and ultraviolet (UVA specifically) light have high energy and cause strong phototoxic reaction [21, $22]$. The phototoxic reaction is primarily induced by exposure of photoreactive chemicals to UV light, and these chemically activated compounds are responsible for the formation of ROS/free radicals [23]. Hence, UV can damage the skin cells, which may lead to aging and cause itchiness, wrinkles, pigmentation, erythema, and eschar formation [21, 22].

This study was conducted to determine antioxidant and hepatoprotective potential of red ginseng extract (RGE) and red ginseng oil (RGO) in mice and the nonphototoxic activity of RGO using the Balb/c 3T3 mouse cell line. Here, we demonstrated that RGE and RGO have potential scavenging effects on free radicals, thus suggesting an important role in oxidative stress.

\section{Materials and Methods}

2.1. Chemicals and Reagents. The following materials were used in this study: RGE and RGO (Korea Ginseng Corporation, Daejeon, Republic of Korea), chlorpromazine hydrochloride (CPZ; Sigma, \#MKBT6268V), Balb/c 3T3 cells, clone A31 (ATCC), DMEM (Gibco, \#2041847), newborn calf serum (Gibco, \#1749272), penicillin/streptomycin (Gibco, \#2019314), neutral red (NR) solution (Sigma, \#RNBG6531), and UV lamp (BIO-SUN, Serial number, 15-101159). All other chemicals and reagents were of the highest grade.

2.2. Sample Preparation of RGE and RGO. Red ginseng powder was obtained from Korea Ginseng Corporation (Daejeon, Republic of Korea). RGE was prepared using water as described in a previously reported procedure with slight modification [24]. Briefly, in hot water, red ginseng was extracted and heated at $90^{\circ} \mathrm{C}$ for $1 \mathrm{~h}$. After cooling, the supernatant was collected and centrifuged at $3000 \mathrm{rpm}$ for $5 \mathrm{~min}$ and evaporated. After evaporation, the samples were stored at $4^{\circ} \mathrm{C}$ until use. RGO was prepared as described previously with modification [8]. Briefly, red ginseng powder was extracted with the $\mathrm{CO}_{2}$ extraction system at a pressure of 450 bar and a temperature of $65^{\circ} \mathrm{C}$. The oil extract of red ginseng was preserved in a vial and stored at $4^{\circ} \mathrm{C}$ until use.

2.3. Cell Culture. Balb/c 3T3 mouse cells were cultured in DMEM supplemented with $10 \%$ newborn calf serum and $1 \% 100 \mathrm{IU} / \mathrm{mL}$ penicillin and $100 \mu \mathrm{g} / \mathrm{mL}$ streptomycin. Cells were seeded in a 96-well plate at different densities for the measurement of cell viability. RGO concentrations of 7.81, $15.63,31.25,62.5,125,500$, and $1000 \mu \mathrm{g} / \mathrm{mL}$ were used. For positive control, CPZ concentrations of $0.04,0.08,0.16$, $0.31,0.63,1.25,2.5$, and $5 \mu \mathrm{g} / \mathrm{mL}$ and $1.56,3.13,6.25,12.5$, $25,50,100$, and $200 \mu \mathrm{g} / \mathrm{mL}$ were used with $\mathrm{UV}$ and without $\mathrm{UV}$, respectively.

2.4. Irradiation Condition. A UV irradiation device (BIOSUN, Serial number, 15-101159) was used for UV light source. The amount of UV light was $5 \mathrm{~J} / \mathrm{cm}^{2}$ with emitting wavelengths of $315-400 \mathrm{~nm}$. For this amount of UV light $\left(5 \mathrm{~J} / \mathrm{cm}^{2}\right)$, the exposure time was calculated by applying the light intensity measured $1 \mathrm{~min}$ after the starting of UV irradiation.

2.5. Animal Treatment. The animals were housed in pathogen-free facility at $21 \pm 2^{\circ} \mathrm{C}$ with a humidity of $60 \pm$ $10 \%$ under a 12-h light and dark cycle and feed and water were supplied ad libitum. Animal care and experimental procedure were conducted with the Institutional Animal Care and Use Committee (IACUC) guidelines, and the animal protocols were approved by the Animal Care Committee of the College of Veterinary Medicine, Kyungpook National University, Daegu, South Korea (approval number: 2019-0046). Male Balb/c mice aged 6 weeks were treated with $100 \mathrm{mg} / \mathrm{kg}$ and $300 \mathrm{mg} / \mathrm{kg}$ of RGE and RGO for 2 weeks orally every day. As a positive control, $75 \mathrm{mg} / \mathrm{kg} \mathrm{N}$-acetyl cysteine (NAC) was used. After 2 weeks, the mice were injected with $400 \mathrm{mg} / \mathrm{kg}$ of APAP through the intraperitoneal route, and after $2 \mathrm{~h}$, the mice were anesthetized, and blood was collected by cardiac puncture. The blood was left to settle for $2 \mathrm{~h}$ in a blood collection tube (BD, Plymouth, UK). The tubes were then centrifuged at $3000 \mathrm{rpm}$ for 15 min to separate the serum, which was collected and stored at $-70^{\circ} \mathrm{C}$ until assay. Liver sample was collected for histopathological examination.

2.6. Measurement of Antioxidant Activity. The DPPH freeradical scavenging activity and $2,2^{\prime}$-azino-bis-3-ethylbenzothiazoline-6-sulfonic acid (ABTS) radical-scavenging activity of RGE and RGO were evaluated using the method described by Kandi Sridhar and Albert Linton Charles with modification [14]. Briefly, RGE and RGO sample extract or positive control (ascorbic acid) with various concentrations were added to the same volume of DPPH $(100 \mu \mathrm{M})$. The mixtures were vortexed and incubated at room temperature for $20 \mathrm{~min}$ 
in the dark. Finally, the absorbance was determined using a spectrophotometer at $515 \mathrm{~nm}$. For the ABTS assay, the ABTS method was produced with reacting the aqueous solution of ABTS with potassium persulfate in the same volume and allowed to react for $24 \mathrm{~h}$ at room temperature in the dark. The ABTS radical cation $(1 \mathrm{~mL})$ solution was mixed with $0.1 \mathrm{~mL}$ of extract or positive control (trolox) at different concentrations. The mixtures were incubated at room temperature for $10 \mathrm{~min}$ in the dark. Finally, the absorbance was measured at $734 \mathrm{~nm}$ with spectrophotometer.

2.7. Measurement of Serum Markers for Oxidative Stress Alanine Aminotransferase and Aspartate Aminotransferase Activity in Serum. Mice were sacrificed $2 \mathrm{~h}$ after the last administration from each group. Blood samples were collected and centrifuged. The levels of alanine aminotransferase (ALT), aspartate aminotransferase (AST), superoxide dismutase (SOD), catalase (CAT), and 8-hydroxy-2-deoxyguanosine (8-OHdG) were measured according to kit instructions.

2.8. Histological Analysis. For evaluating the histopathological changes, the liver tissues were fixed in $10 \%$ formalin and routinely processed in a graded ethanol series and toluene, as described previously [25]. The tissues were then embedded in paraffin and sectioned into $5-\mu \mathrm{m}$-thick slices. The sections were stained with hematoxylin and eosin (H\&E) as previously described, and the pathological changes were observed under a light microscope.

2.9. Determination of Phototoxicity. Phototoxicity was evaluated using RGO and CPZ on the mouse fibroblast cell line Balb/c 3T3 using a previously described method [21]. Cells were seeded in two 96-well plates with (+UV) and without (-UV) UV light for RGO and CPZ at a density of $1 \times 10^{4}$ cells/well. DMSO in cell media was used as a negative control. After dispensing $100 \mu \mathrm{L}$ of cell suspension $\left(1 \times 10^{4}\right.$ cells/well $)$ in the culture medium, the plates were incubated for $24 \mathrm{~h}$ at $37^{\circ} \mathrm{C}$ and $5 \% \mathrm{CO}_{2}$. After $24 \mathrm{~h}$, the medium was removed, cells were washed with PBS, and $100 \mu \mathrm{L}$ of the test materials, positive control, and negative control were added to the wells $(n=3)$. The plates were incubated at $37^{\circ} \mathrm{C}$ and $5 \% \mathrm{CO}_{2}$ for $1 \mathrm{~h}$, after which the irradiated plate was exposed to UV light $\left(5 \mathrm{~J} / \mathrm{cm}^{2}\right)$, and the nonirradiated plate was shielded with an aluminum foil. After UV exposure, the medium was decanted, and $100 \mu \mathrm{L}$ of the medium was added. The cells were incubated at $37^{\circ} \mathrm{C}$ and $5 \% \mathrm{CO}_{2}$ for $24 \mathrm{~h}$. The medium was removed and washed with PBS. Then, $100 \mu \mathrm{L}$ of NR solution $(50 \mu \mathrm{g} / \mathrm{mL})$ was added to each well and incubated at $37^{\circ} \mathrm{C}$ and $5 \% \mathrm{CO}_{2}$ for $3 \mathrm{~h}$. After removing the NR medium, it was washed with PBS. Next, $150 \mu \mathrm{L}$ of NR extractant solution was added to each well, and the plates were shaken on a plate shaker for $30 \mathrm{~min}$ in a light-shielded state. Finally, the absorbance was measured at a wavelength of $540 \mathrm{~nm}$ using a multichannel microplate reader. The absorbance data were used in the phototox software (Phototox 2.0, ZEBET at the BFR, Berlin Germany) to obtain cell viability data at different concentrations.
2.10. Statistical Analysis. The experimental data were expressed as mean \pm the standard deviation (SD). Statistical significance was determined using ANOVA. The statistical significance of data is denoted on the graphs by asterisks $\left({ }^{*}\right)$, with the significance values as ${ }^{*} P<0.05,{ }^{* *} P<0.01$, and ${ }^{* * *} P<0.001$.

\section{Results}

3.1. Effects of RGE and RGO on DPPH and ABTS Free Radicals. The antioxidant activities of RGE and RGO were determined using the DPPH and ABTS assay. The results showed that RGE effectively scavenged the radicals of $\mathrm{DPPH}$ at $60 \mathrm{mg} / \mathrm{mL}$ to the extent of the positive control, which was $10 \mathrm{mg} / \mathrm{mL}$ of ascorbic acid (Figure 1(a)). On the other hand, RGO scavenged the radicals of DPPH from concentrations of 125 to $500 \mathrm{mg} / \mathrm{mL}$ (Figure 1(b)). Regarding the ABTS assay results, it was found that RGE effectively reduced the radicals completely at $5 \mathrm{mg} / \mathrm{mL}$ concentration, similar to the level of trolox, a water-soluble conjugate of vitamin $\mathrm{E}$ that was used as a positive control in this assay (Figure 1(c)). The concentration of trolox used in this study was $10 \mu \mathrm{M}$. In the case of RGO, a concentration of 30 $\mathrm{mg} / \mathrm{mL}$ showed an efficacy of approximately $80 \%$ in inhibiting ABTS radicals (Figure $1(\mathrm{~d})$ ).

3.2. Effects of RGE and RGO on ALT and AST Activity in Serum. APAP treatment resulted in extremely elevated levels of ALT in the serum of mice compared to the control group. However, this increase was significantly reduced by NAC treatment. Treatment with RGE and RGO also reduced the levels of ALT in a dose-dependent manner (Figure 2(a)). NAC treatment reduced the levels of AST in serum induced by APAP. Treatment with $100 \mathrm{mg} / \mathrm{kg}$ RGE did not significantly reduce the levels of AST. However, treatment with $300 \mathrm{mg} / \mathrm{kg}$ RGE significantly reduced the levels of AST (Figure 2(b)). Comparatively, both concentrations of RGE and RGO led to a significant reduce in ALT and AST levels comparable to the levels observed with NAC treatment.

3.3. Effects of RGE and RGO on Oxidative Stress in Serum. $\mathrm{SOD}$ is an enzyme known to catalyze dismutation of the superoxide $\left(\mathrm{O}_{2}{ }^{-}\right)$radical into oxygen and hydrogen peroxide, which comprises an extremely important defense against oxidative stress in the body. Our results showed that APAP considerably reduced the levels of SOD in the serum. This reduction was recovered by NAC treatment. Treatment with $100 \mathrm{mg} / \mathrm{kg}$ of both RGO and RGE showed no significant increase in SOD levels. However, high doses of both RGO and RGE led to recovery of SOD levels (Figure 2(c)). CAT is an enzyme that is detected in all organisms when exposed to oxygen as it catalyzes the decomposition of hydrogen peroxide to water and oxygen. Therefore, it is an important enzyme against oxidative stress. Our study results showed that APAP reduced the activity of CAT compared to that in the control group. NAC treatment improved CAT levels in the serum to even higher levels than those in the control group. Both RGO and RGE increased the CAT activity in a dose-dependent manner (Figure 2(d)). 8-OHdG is the major 


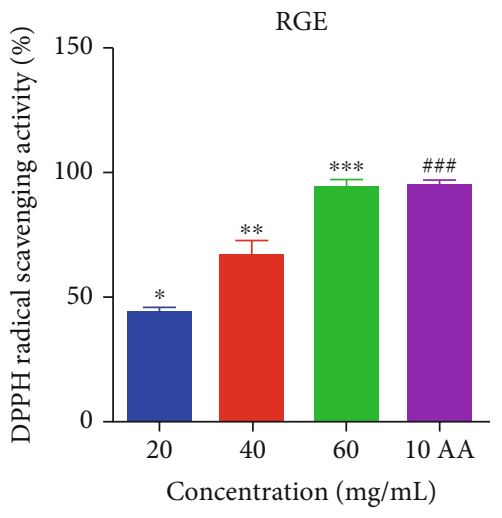

(a)

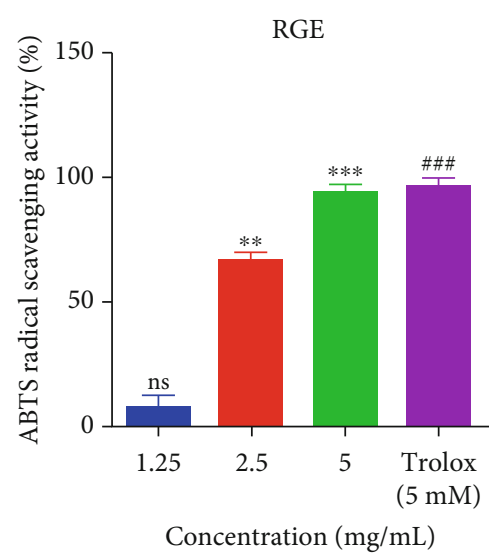

(c)

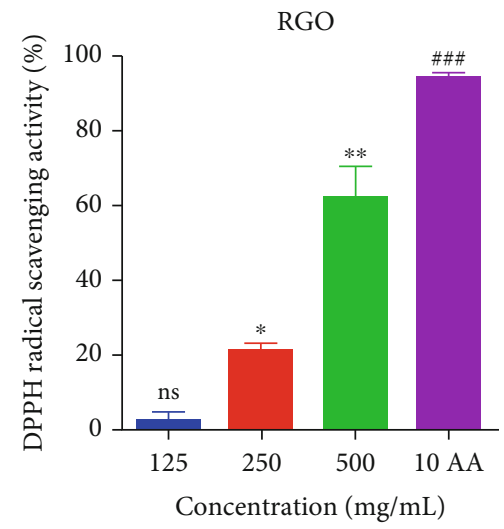

(b)

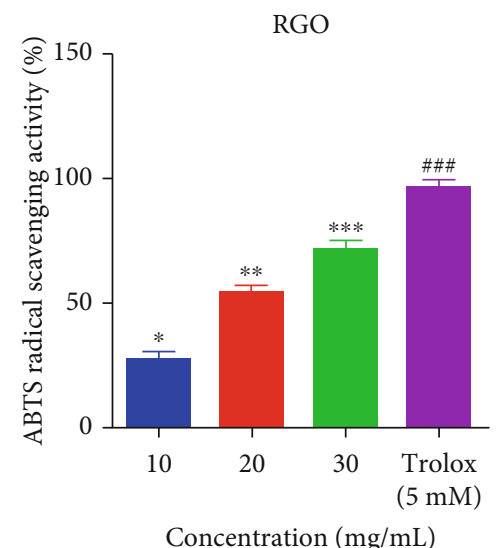

(d)

FIGURE 1: Determination of in vitro antioxidant activities of red ginseng extract (RGE) and red ginseng oil (RGO). (a, b) DPPH and (c, d) ABTS assays for RGE and RGO, respectively. In DPPH and ABTS methods, the standards were ascorbic acid (AA) in DPPH and trolox in ABTS, respectively. Radical-scavenging capacities were dose-dependent upon treatment with RGE and RGO. Data are presented as mean \pm standard deviation (SD) $(n=3) .{ }^{\# \#} P<0.001$ for standard, ${ }^{*} P<0.05,{ }^{* *} P<0.01$, and ${ }^{* * *} P<0.001$ compared with standard.

product of DNA oxidation and is a marker of DNA damage. Our results demonstrated that in mice treated with APAP, 8OHdG activity was increased and improved with NAC treatment. Treatment with RGE and RGO also improved the activity of $8-\mathrm{OHdG}$ in a dose-dependent manner, where treatment with $300 \mathrm{mg} / \mathrm{kg}$ of both samples recovered the DNA oxidative damage to the levels of the control group (Figure 2(e)). These results may be due to the presence of the major ginsenosides in red ginseng determined by ultraperformance liquid chromatography (UPLC) (Table 1).

3.4. Effects of RGE and RGO on Pathological Changes in the Liver. Hepatocyte cells showed normal morphology, the hepatic lobules and sinusoids were intact, and the central veins were normal in the control group. However, in the APAP-treated group, the central veins were congestive and dilated, and degeneration of hepatocytes, infiltration of inflammatory cells, vacuolation of hepatocytes, and necrosis of hepatocytes were observed. In the NAC-treated group, there were no histopathological changes. In addition, treatment with $100 \mathrm{mg} / \mathrm{kg}$ of RGE and RGO significantly recovered the tissue damages compared to the APAPtreated group. Moreover, groups treated with $300 \mathrm{mg} / \mathrm{kg}$ of
RGE and RGO showed normal arrangement of hepatocytes with no significant damage and infiltration of inflammatory cells and hepatocytes, similar to that in the control group (Figure 3). This result indicated that $\mathrm{RGE}$ and RGO have significant protective effects against APAPinduced hepatic injury.

3.5. Effects of RGO on Phototoxicity. Phototoxicity was evaluated using RGO and CPZ in Balb/c 3T3 mouse cell line. Cell viability was measured at various concentrations of RGO and $\mathrm{CPZ}$ in the presence (exposure) or absence (no exposure) of UV light (Figures 4(a)-4(d)). RGO was found to be nonphototoxic and displayed insensitivity to phototoxic reaction (Figure 5(a)), whereas CPZ demonstrated toxicity potential in the presence of UV light (Figure 5(b)). Earlier, Peters and Holzhutter (2002) reported that sample is considered to be nonphototoxic if it has a photoirritation factor (PIF) $<2$ or a mean photo effect $(\mathrm{MPE})<0.1$; probably phototoxic if $\mathrm{PIF}$ is $>2$ and $<5$ or MPE is $>0.1$ and $<0.15$; and phototoxic if $\mathrm{PIF}$ is $>5$ or MPE is $>0.15$. In the case of CPZ, the PIF and MPE values were 25.721 and 0.236 , respectively. Based on the assessment of parameters, RGO was found to be completely nonphototoxic, with the MPE being 0.006 (MPE $<0.1$, 


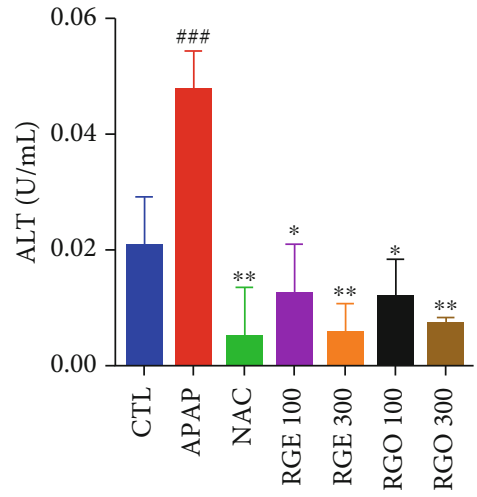

(a)

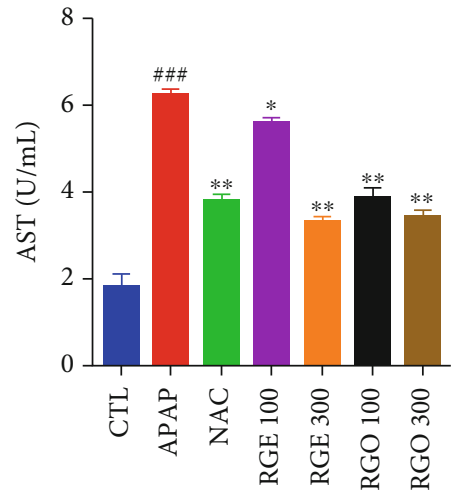

(b)

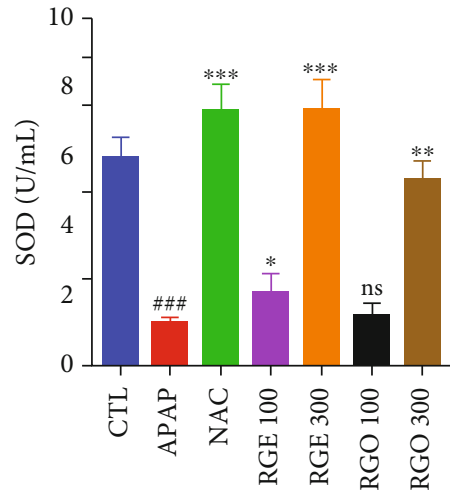

(c)

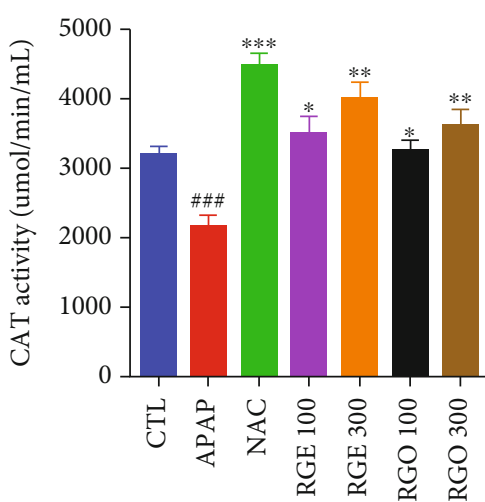

(d)

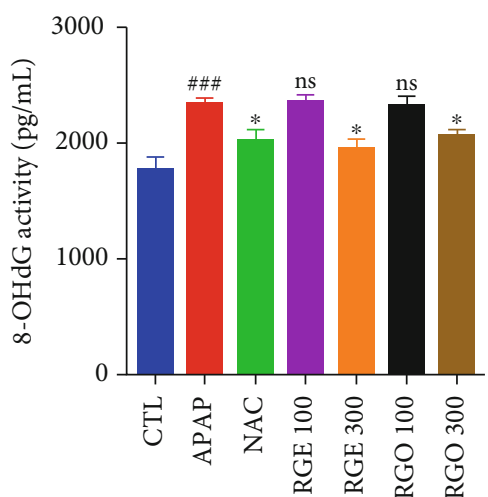

(e)

FIGURE 2: Effects of RGE and RGO in serum markers on APAP-induced liver injury in mice. (a) Effects of RGE and RGO on the ALT level in the serum. (b) Effects of RGE and RGO on the AST level in the serum. (c) Effects of RGE and RGO on the SOD activity in the serum. (d) Effects of RGE and RGO on the CAT activity in the serum. (e) Effects of RGE and RGO on the 8-OHdG content in the serum. Data are presented as mean \pm standard deviation $(\mathrm{SD})(n=5)$. Significant difference at ${ }^{\# \# \#} P<0.001$ compared with control (CTL) group and ${ }^{*} P<0.05,{ }^{* *} P<0.01$, and ${ }^{* * *} P<0.001$ compared with the APAP-treated group.

nonphototoxic) [26]. In contrast, CPZ exhibited phototoxic activity, with the MPE being 0.236 (MPE > 0.15, phototoxic), in the presence of UV light (Table 2).

\section{Discussion}

The liver is the major organ that metabolizes the majority of drugs. APAP is one of the most widely used drugs to reduce fever and pain. However, an overdose of APAP induces oxidative stress and production of ROS, which causes metabolic dysfunction, damage to the antioxidant defense system, and other tissue and hepatic injuries [20,27]. Similarly, when cells are continuously exposed to sunlight, free radicals such as superoxide anion and hydroxyl radical and nonradical intermediates, including hydrogen peroxide and singlet oxygen, are generated [21]. These ROS can be produced by several sources, such as water, molecular oxygen, and enzymes. However, cells react to these ROS using nonenzymatic antioxidants such as vitamin C, vitamin E, and enzymatic antioxidants such as CAT and SOD [28]. Imbalance between these systems can modulate protein function, causing destructive action on DNA, which has been implicated in mutagenesis, carcinogenesis, and aging $[21,29]$. Therefore, the inhibition of oxidative stress is a promising therapeutic strategy for liver damage and phototoxicity.
TABLE 1: Determination of ginsenosides in red ginseng [1]. Data are presented as mean \pm standard deviation (SD) $(n=3)$.

\begin{tabular}{lcc}
\hline Classification & Ginsenoside & Composition $(\mathrm{mg} / \mathrm{g})$ \\
\hline \multirow{3}{*}{ Panaxatriol } & Rg1 & $3.00 \pm 0.033$ \\
& Rg2 & $3.26 \pm 0.085$ \\
& Rg3 & $4.04 \pm 0.049$ \\
\hline \multirow{3}{*}{ Panaxadiol } & Rb1 & $10.51 \pm 0.048$ \\
& Rb2 & $4.51 \pm 0.053$ \\
& Rb3 & $0.32 \pm 0.067$ \\
& Rc & $5.37 \pm 0.074$ \\
& Rd & $3.47 \pm 0.111$ \\
& Rk1 & $4.04 \pm 0.051$ \\
\hline
\end{tabular}

Several studies have demonstrated that natural antioxidants have potential pharmacological activities to protect organs from toxic substances, including the adverse effects of APAP and sunlight. The major active components in the RGE are the ginsenosides which play key role for the various beneficial effects [1]. Previous study demonstrated that sitosterol was influenced the cellular protective systems via 

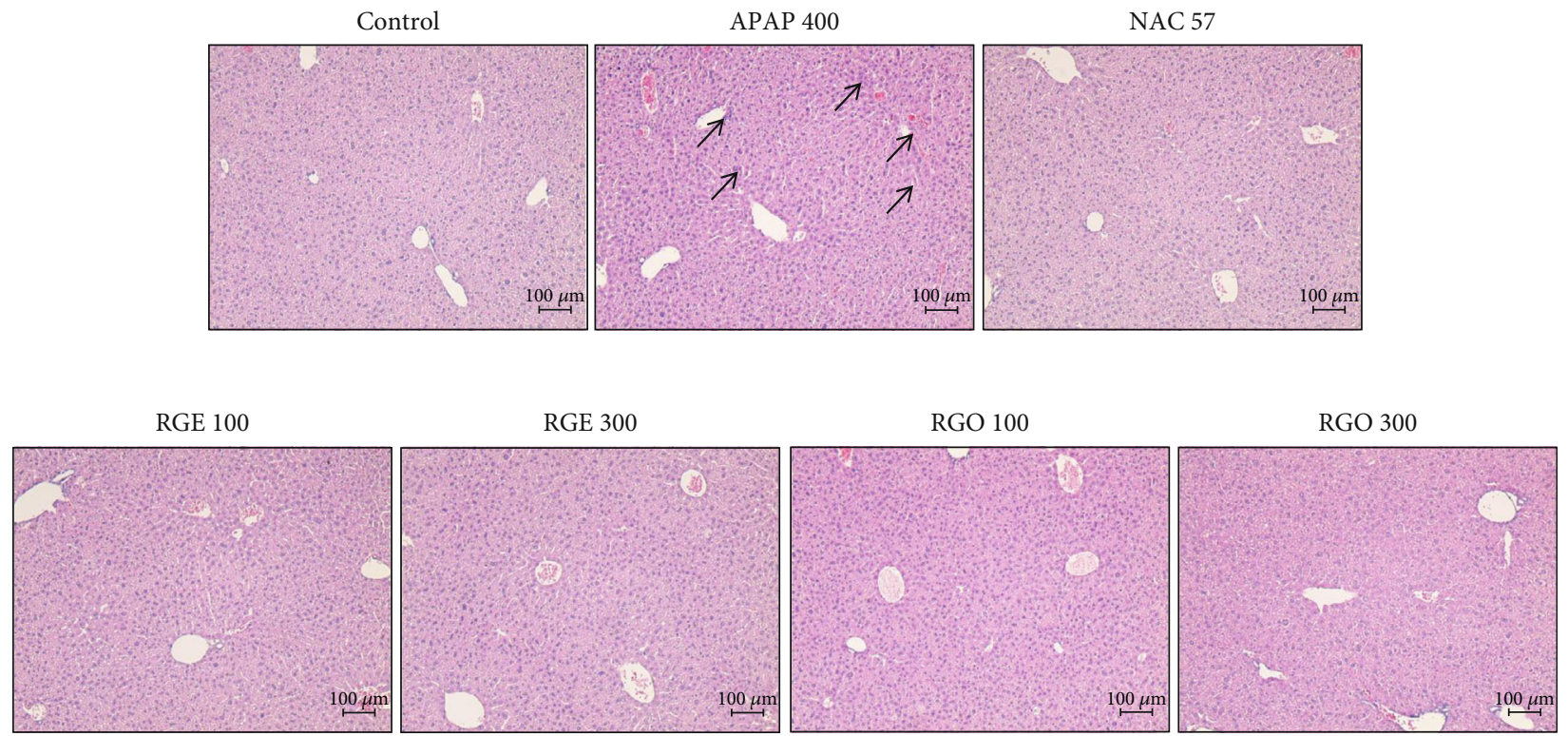

FIGURE 3: Histopathological changes by RGE and RGO on APAP-induced liver injury in mice. Mice were treated with APAP (400 mg/kg) to induce hepatotoxicity. In the APAP-treated group, there were degeneration of hepatocytes, congestive and dilated central veins, infiltration of inflammatory cells, vacuolation of hepatocytes, and necrosis of hepatocytes (arrows). N-acetyl cysteine (75 mg/kg) was used as a positive control. The concentrations of 100 and $300 \mathrm{mg} / \mathrm{mL}$ were used for RGE and RGO, respectively. Original magnification $\times 100$.

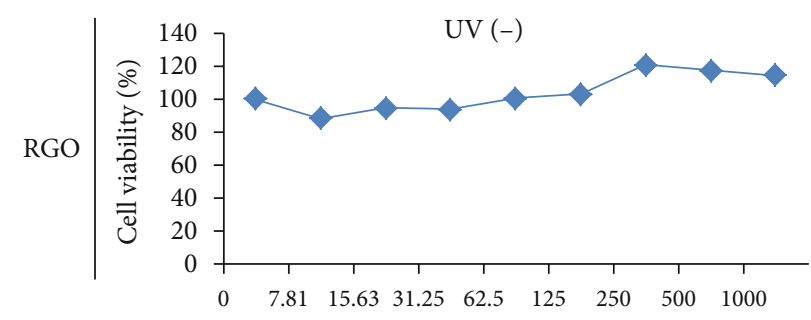

(a)

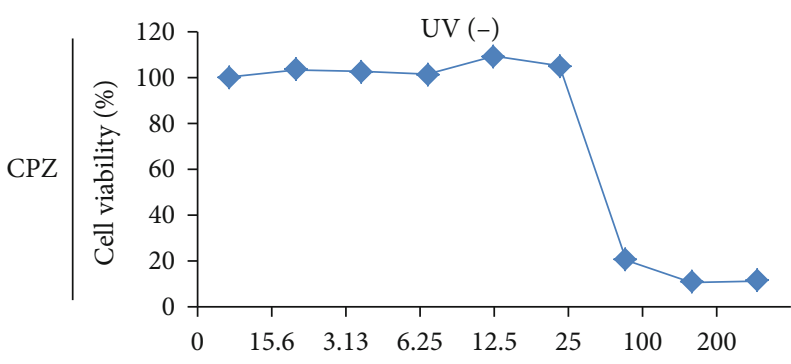

(c)

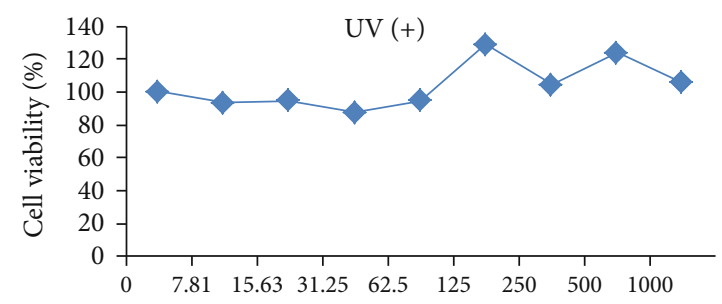

(b)

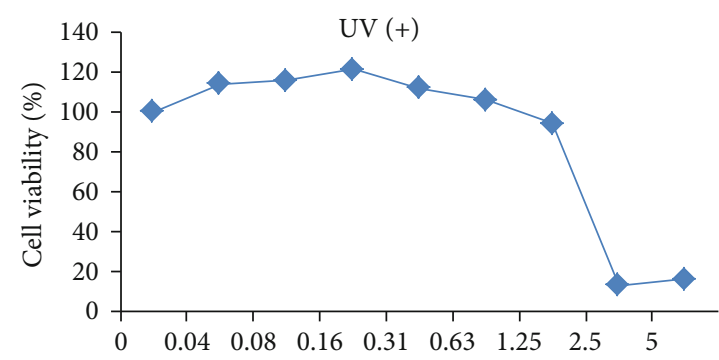

(d)

FIGURE 4: Effects of RGO and CPZ on cell viability with UV (-) and UV (+) light exposure in Balb/c 3T3 cells. (a, b) Cell viability was evaluated at different concentrations of RGO in the absence or presence of UV light. (c, d) Cell viability was evaluated at different concentrations of chlorpromazine (CPZ) in the absence or presence of UV light.

enhancement of nuclear factor erythroid-2 related factor-2 (Nfr2) pathway in HepG2 cells [30]. Moreover, betasitosterol and stigmasterol were protected oxidative stress induced by nitrophenol through activation of Nrf2-mediated antioxidant enzymes in rat, reported by Zhang et al. [31].

In this study, the antioxidant properties of RGE and RGO were investigated using the commonly used antioxidant methods, including DPPH and ABTS assays. The results clearly demonstrated that both RGE and RGO significantly inhibited the production of free radicals based on the concentration obtained by DPPH and ABTS methods (Figures 1(a)$1(d))$. The scavenging capacity of DPPH increased in a dosedependent manner $(20,40$, and $60 \mathrm{mg} / \mathrm{mL}$ and 125,150 , and $500 \mathrm{mg} / \mathrm{mL}$ for RGE and RGO, respectively). Ascorbic acid was used as a standard solution $(10 \mathrm{mg} / \mathrm{mL})$ that exhibited the highest inhibition (95.25\%) by the DPPH method, whereas the highest inhibition value was $94.13 \%$ for RGE at the concentration $60 \mathrm{mg} / \mathrm{mL}$. Similarly, with RGO treatment, 


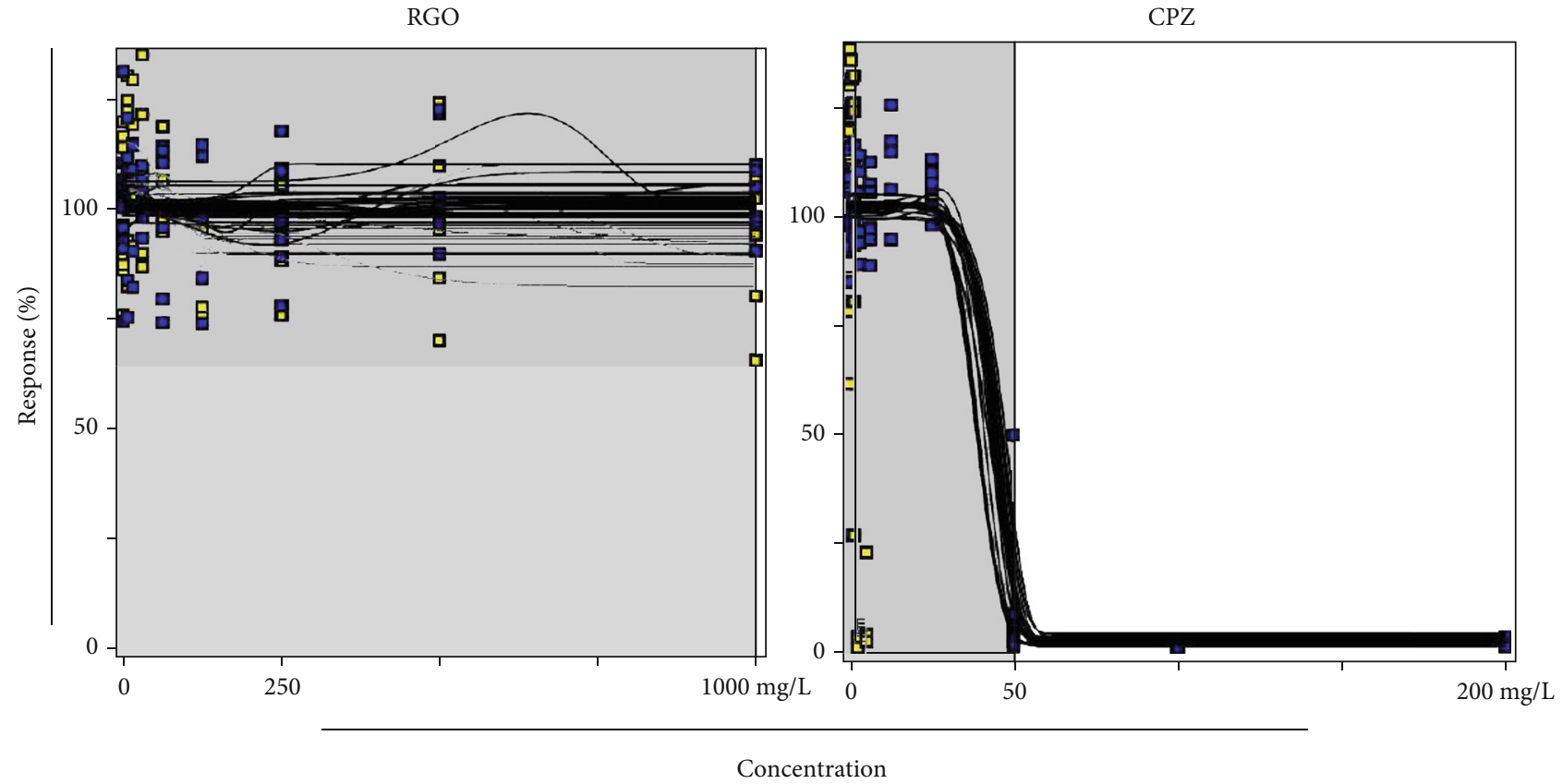

$\square: \mathrm{UV}(-)$
$\square: \mathrm{UV}(+)$

FIGURE 5: Effects of concentrations in RGO and CPZ with UV (-) and UV (+) light exposure in Balb/c 3T3 cells. (a) Effect of increasing concentrations with UV (-) and UV (+) of RGO on percentage response of Balb/c 3T3 cells. (b) Effect of increasing concentrations with UV (-) and UV (+) of CPZ on percentage response of Balb/c 3T3 cells. No exposure of UV light (blue) and exposure to UV light (yellow).

TABLE 2: Using the software (Phototox 2.0, at the BFR, Berlin Germany), photo irritation factor (PIF), and mean photo effect (MPE) values were generated for RGO and $\mathrm{CPZ}$ in Balb/c $3 \mathrm{~T} 3$ cells.

\begin{tabular}{lcccc}
\hline Group & PIF & MPE & Phototoxicity & Phototoxic parameter \\
\hline RGO & 0 & 0.006 & Nonphototoxic & PIF $<2$ or MPE $<0.1=$ nonphototoxic \\
CPZ & 25.721 & 0.236 & Phototoxic & PIF $>2$ and $<5$ or MPE $>0.1$ and $<0.15=$ probably phototoxic \\
& & & PIF $>5$ or MPE $>0.15=$ phototoxic \\
\hline
\end{tabular}

the maximum inhibition was $62.64 \%$ at the highest concentration of $500 \mathrm{mg} / \mathrm{mL}$. The DPPH scavenging capacity results showed that RGE and RGO exhibited high antioxidant effects compared with ascorbic acid. Ryu et al. reported about the free radical-scavenging capacity of Korean red ginseng for erectile dysfunction in rats with noninsulin-dependent diabetes mellitus [5].

On the other hand, a similar trend was observed by the ABTS method, wherein the scavenging activity of ABTS was increased in a dose-dependent manner (1.25, 2.5, and $5 \mathrm{mg} / \mathrm{mL}$ and 10,20 , and $30 \mathrm{mg} / \mathrm{mL}$ for RGE and RGO, respectively). This result was compared with the standard solution trolox $(5 \mathrm{mM})$, a vitamin E conjugate, which showed the highest inhibition (97.25\%). RGE treatment resulted in the highest inhibition value (95.14\%) at the highest concentration $(5 \mathrm{mg} / \mathrm{mL})$, whereas RGO treatment also exhibited the same trend of the highest inhibition (72.7\%) at the highest concentration $(30 \mathrm{mg} / \mathrm{mL})$. This result indicated that both RGE and RGO possess potent antioxidant capacity as evaluated by the ABTS assay. Earlier report demonstrated that ethanolic extracts of red ginseng and puffed red ginseng significantly showed radical scavenging ability by DPPH and ABTS assays [3].

Liver enzymes, primarily ALT and AST, in the blood are the major indicators to evaluate liver function [32]. Increasing levels of serum liver enzymes indicate hepatic injury because these enzymes are normally located in the cytoplasm, but after hepatic dysfunction, the enzymes are released into the circulation. An overdose of APAP increases the levels of liver enzymes, indicating liver damage. Kim et al., reported that pretreatment with Korean red ginseng reduced the expression of ALT and AST levels on aflatoxin B1-induced liver toxicity in rat model [33]. Our results demonstrated that serum ALT and AST levels were significantly reduced by RGE and RGO treatment (Figures 2(a) and 2(b)), suggesting that both RGE and RGO have protective activity against the APAP-induced liver injury.

SOD and CAT are the most important antioxidant enzymes against ROS. Under the normal physiological condition, SOD and CAT counteract with free radicals, but these 


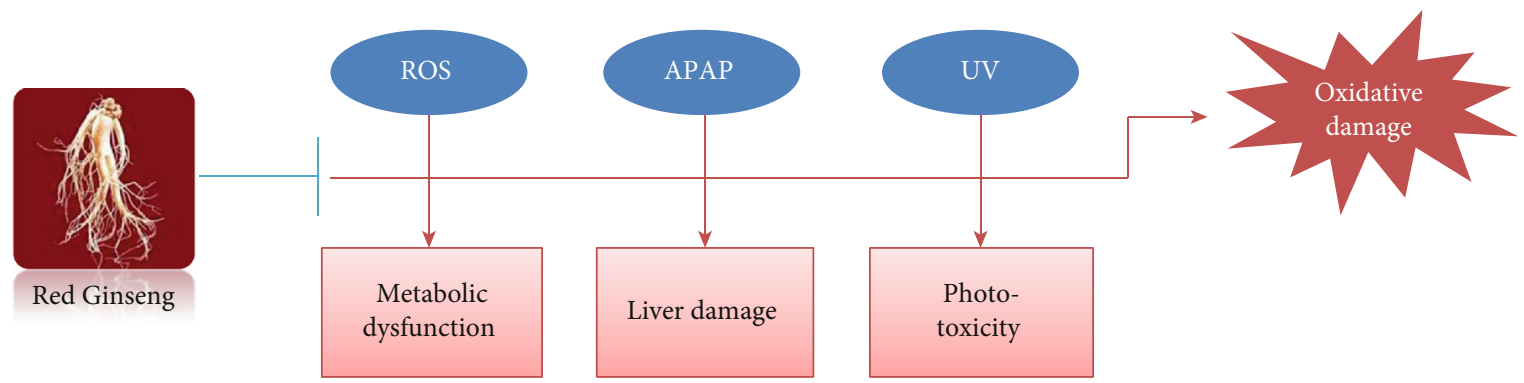

FIGURE 6: Schematic model showing the role of red ginseng in oxidative damage. Free radicals, overdose of acetaminophen (APAP), and UV radiation lead to metabolic dysfunction, liver toxicity, and phototoxicity, causing damage to the antioxidant defense system. Red ginseng extract (RGE) and red ginseng oil (RGO) protect against reactive oxygen species (ROS) and APAP- and UV-induced oxidative damage.

enzymes are reduced when there is overproduction of ROS [34-36]. In the past, it was reported that saponin fraction of red ginseng supplementation was markedly increased SOD and catalase levels in hepatic tissue in mice [37]. In this study, the levels of SOD and CAT were significantly reduced after APAP administration; meanwhile, RGE and RGO treatment significantly elevated their activities (Figures 2(c) and $2(d)$ ). These results indicated that both RGE and RGO significantly elevated the levels of SOD and CAT in mice serum. Our results supported the previous report which stated that RGE significantly upregulated the SOD activity in healthy subjects (age 20-65 years) [38].

$8-\mathrm{OHdG}$ is the major product of DNA oxidation, and its elevation in the serum indicates excessive oxidative stress [34, 39]. An overdose of APAP increased the DNA oxidation and reduced the activity of antioxidant enzymes. Our results demonstrated that the levels of 8-OHdG were significantly reduced in the RGE- and RGO-treated groups (Figure 2(e)), suggesting that both RGE and RGO could protect DNA oxidation and improve the DNA damage induced by APAP. Red ginseng extract showed potential antioxidant activity in oxidative stress-mediated DNA damage, various studies previously reported $[33,40]$.

Liver histological pathology is another indicator to evaluate hepatic injury $[32,41]$. Observation of the hepatic tissue histopathology confirmed the inhibitory effects of RGE and RGO in APAP-treated liver damage. According to the H\&E staining result, the RGE- and RGO-treated groups showed reduced hepatocyte degeneration and decreased infiltration of inflammatory cells compared to the APAP-treated group (Figure 3). Moreover, significantly reduced hepatic necrosis and dilated sinusoids were observed in the RGE- and RGOtreated groups. This protective activity was dose-dependent, although there was no high difference between two doses $(100$ and $300 \mathrm{mg} / \mathrm{mL})$ in these groups. Based on histopathological findings, there was no significant difference between NAC treatment at $75 \mathrm{mg} / \mathrm{mL}$ and the control group. Pretreated with Korean red ginseng were significantly suppressed hepatic lesions such as necrosis of hepatocytes, hemorrhage, and loss of hepatic cords according to previous report [33]. Our results demonstrated that both RGE and RGO have significant protective capacity against APAPinduced hepatic damage.

In this study, we demonstrated RGO provides protection against UV light in Balb/c 3T3 cells (Figure 5(a)), suggesting that RGO has nonphototoxic capacity. It is assumed that the nonphototoxic activity could be caused because of antioxidant properties. CPZ was used as a positive control due to the phototoxic activity (Figure 5(b)) and caused damage to the DNA by direct and indirect mechanisms [21, 42]. However, further studies are required to confirm the degree of nonphototoxic activity of RGO.

\section{Conclusions}

In summary, RGE and RGO exhibited antioxidant and radical-scavenging properties, suggesting that both RGE and RGO possessing antioxidant activities can prevent the cell or tissue from oxidative damage induced by toxic chemicals or radiation (Figure 6). Our study indicated that Korean red ginseng may be potential substance in skin care and sunscreen formulations. To the best of our knowledge, this is the first report to demonstrate the effects of RGE and RGO on oxidative stress and attenuated activity of RGO on ultraviolet-induced photo toxicity. Further study in large scale is mandatory to determine the exact mechanism underlying the protective effects of red ginseng.

\section{Data Availability}

Data analyzed or generated during this study are included in this manuscript.

\section{Conflicts of Interest}

The authors declare that they have no competing interest.

\section{Authors' Contributions}

H M Arif Ullah, Yuan Yee Lee, and Mansour Abdullah Sandhu contributed equally to this work.

\section{Acknowledgments}

This study was supported by the grant from the Korean Society of Ginseng (2018).

\section{References}

[1] E. Saba, Y. Y. Lee, M. Kim et al., “A novel herbal formulation consisting of red ginseng extract and Epimedium koreanum 
Nakai-attenuated dextran sulfate sodium-induced colitis in mice," Journal of Ginseng Research, vol. 44, no. 6, pp. 833842, 2020.

[2] S. Lee, K. Youn, W.-S. Jeong, C.-T. Ho, and M. Jun, "Protective effects of red ginseng oil against $\mathrm{A} \beta_{25}{ }_{35}$-induced neuronal apoptosis and inflammation in PC12 cells," International Journal of Molecular Sciences, vol. 18, no. 10, p. 2218, 2017.

[3] S.-J. Lee, S. Oh, M.-J. Kim, G.-S. Sim, T. W. Moon, and J. Lee, "Oxidative stability of extracts from red ginseng and puffed red ginseng in bulk oil or oil-in-water emulsion matrix," Journal of ginseng research, vol. 42, no. 3, pp. 320-326, 2018.

[4] H. Liu, J. Wang, M. Liu et al., "Antiobesity effects of ginsenoside Rg1 on 3T3-L1 preadipocytes and high fat diet-induced obese mice mediated by AMPK," Nutrients, vol. 10, no. 7 , p. 830, 2018.

[5] J.-K. Ryu, T. Lee, D.-J. Kim et al., "Free radical-scavenging activity of Korean red ginseng for erectile dysfunction in non-insulin-dependent diabetes mellitus rats," Urology, vol. 65, no. 3, pp. 611-615, 2005.

[6] J. Kim, "Protective effects of Asian dietary items on cancerssoy and ginseng," Asian Pacific Journal of Cancer Prevention, vol. 9, no. 4, pp. 543-548, 2008.

[7] M. Irfan, M. Kim, and M. H. Rhee, "Anti-platelet role of Korean ginseng and ginsenosides in cardiovascular diseases," Journal of ginseng research, vol. 44, no. 1, pp. 24-32, 2020.

[8] M.-J. Bak, M. Jun, and W.-S. Jeong, "Antioxidant and hepatoprotective effects of the red ginseng essential oil in $\mathrm{H}_{2} \mathrm{O}_{2}$ treated HepG2 cells and $\mathrm{CCl}_{4}$-treated mice," International Journal of Molecular Sciences, vol. 13, no. 2, pp. 2314-2330, 2012.

[9] J.-M. Lu, Q. Yao, and C. Chen, "Ginseng compounds: an update on their molecular mechanisms and medical applications," Current Vascular Pharmacology, vol. 7, no. 3, pp. 293-302, 2009.

[10] S. M. Lee, B.-S. Bae, H.-W. Park et al., "Characterization of Korean Red Ginseng (Panax ginseng Meyer): history, preparation method, and chemical composition," Journal of ginseng research, vol. 39, no. 4, pp. 384-391, 2015.

[11] A. W. B. Reyes, H. T. Hop, L. T. Arayan et al., "The host immune enhancing agent Korean red ginseng oil successfully attenuates Brucella abortus infection in a murine model," Journal of Ethnopharmacology, vol. 198, pp. 5-14, 2017.

[12] M. J. Bak, V.-L. Truong, S.-Y. Ko et al., "Induction of Nrf2/ARE-mediated cytoprotective genes by red ginseng oil through ASK1-MKK4/7-JNK and p38 MAPK signaling pathways in HepG2 cells," Journal of ginseng research, vol. 40, no. 4, pp. 423-430, 2016.

[13] E. Saba, S.-H. Kim, Y. Y. Lee et al., “Anti-melanogenic effects of Korean red ginseng oil in an ultraviolet B-induced hairless mouse model," Molecules, vol. 25, no. 20, p. 4755, 2020.

[14] K. Sridhar and A. L. Charles, "In vitro antioxidant activity of Kyoho grape extracts in DPPH and ABTS assays: estimation methods for $\mathrm{EC}_{50}$ using advanced statistical programs," Food Chemistry, vol. 275, pp. 41-49, 2019.

[15] R. F. de Araújo, D. B. G. Martins, and M. A. C. Borba, "Oxidative stress and disease," in A master regulator of oxidative stress-the transcription factor nrf2, IntechOpen, 2016.

[16] P. Sharma, A. B. Jha, R. S. Dubey, and M. Pessarakli, "Reactive oxygen species, oxidative damage, and antioxidative defense mechanism in plants under stressful conditions," Journal of botany, vol. 2012, Article ID 217037, 26 pages, 2012.
[17] A. J. Makin and R. Williams, "Acetaminophen-induced hepatotoxicity: predisposing factors and treatments," Advances in Internal Medicine, vol. 42, pp. 453-483, 1997.

[18] W. M. Lee, "Drug-induced hepatotoxicity," The New England Journal of Medicine, vol. 349, no. 5, pp. 474-485, 2003.

[19] M. R. McGill, C. D. Williams, Y. Xie, A. Ramachandran, and H. Jaeschke, "Acetaminophen-induced liver injury in rats and mice: comparison of protein adducts, mitochondrial dysfunction, and oxidative stress in the mechanism of toxicity," Toxicology and Applied Pharmacology, vol. 264, no. 3, pp. 387-394, 2012.

[20] H. Nikravesh, M. J. Khodayar, M. Mahdavinia, E. Mansouri, L. Zeidooni, and F. Dehbashi, "Protective effect of gemfibrozil on hepatotoxicity induced by acetaminophen in mice: the importance of oxidative stress suppression," Advanced pharmaceutical bulletin, vol. 8, no. 2, pp. 331-339, 2018.

[21] V. Rai, N. Dayan, and B. Michniak-Kohn, "A comparative evaluation of photo-toxic effect of fractionated melanin and chlorpromazine hydrochloride on human (dermal fibroblasts and epidermal keratinocytes) and mouse cell line/s (fibroblast Balb/c 3T3)," Toxicology In Vitro, vol. 25, no. 2, pp. 538-544, 2011.

[22] N. H. Youn, E. J. Kim, J.-S. Yi et al., "Evaluation of skin phototoxicity of transdermally administered pharmaceuticals in Sprague-Dawley rats," Laboratory Animal Research, vol. 36, no. 1, pp. 42-47, 2020.

[23] Y. Yonezawa, T. Ohsumi, T. Miyashita et al., "Evaluation of skin phototoxicity study using SD rats by transdermal and oral administration," The Journal of toxicological sciences, vol. 40, no. 6, pp. 667-683, 2015.

[24] T. Ramesh, S.-W. Kim, S.-Y. Hwang, S.-H. Sohn, S.-K. Yoo, and S.-K. Kim, "Panax ginseng reduces oxidative stress and restores antioxidant capacity in aged rats," Nutrition Research, vol. 32, no. 9, pp. 718-726, 2012.

[25] Y.-S. Son, H. M. A. Ullah, A. K. Elfadl et al., "Preventive effects of vitamin $\mathrm{C}$ on diethylnitrosamine-induced hepatotoxicity in Smp 30 knockout mice," In Vivo, vol. 32, no. 1, pp. 93-99, 2018.

[26] B. Peters and H.-G. Holzhütter, "In vitro phototoxicity testing: development and validation of a new concentration response analysis software and biostatistical analyses related to the use of various prediction models," Alternatives to Laboratory Animals, vol. 30, no. 4, pp. 415-432, 2002.

[27] M. Y. Yoon, S. J. Kim, B.-H. Lee, J.-H. Chung, and Y. C. Kim, "Effects of dimethylsulfoxide on metabolism and toxicity of acetaminophen in mice," Biological \& Pharmaceutical Bulletin, vol. 29, no. 8, pp. 1618-1624, 2006.

[28] K. Brawn and I. Fridovich, "Superoxide radical and superoxide dismutases: threat and defense," in Autoxidation in food and biological systems, pp. 429-446, Springer, 1980.

[29] P. Fu, S.-H. Cheng, L. Coop et al., "Photoreaction, phototoxicity, and photocarcinogenicity of retinoids," Journal of Environmental Science and Health, Part C, vol. 21, no. 2, pp. 165-197, 2003.

[30] H. S. Kang, M. J. Park, K. S. Jin et al., "Regulatory roles of Chrysanthemum zawadskii roots in nuclear factor E2-related factor 2/antioxidant response element pathway," Food Science and Biotechnology, vol. 17, no. 2, pp. 367-372, 2008.

[31] Y. Zhang, M. Song, X. Rui, S. Pu, Y. Li, and C. Li, "Supplemental dietary phytosterin protects against 4-nitrophenol-induced oxidative stress and apoptosis in rat testes," Toxicology reports, vol. 2, pp. 664-676, 2015. 
[32] Y. Zhuang, Q. Ma, Y. Guo, and L. Sun, "Protective effects of rambutan (Nephelium lappaceum) peel phenolics on $\mathrm{H}_{2} \mathrm{O}_{2}$ induced oxidative damages in HepG2 cells and d-galactoseinduced aging mice," Food and Chemical Toxicology, vol. 108, no. Part B, pp. 554-562, 2017.

[33] Y.-S. Kim, Y.-H. Kim, J.-R. Noh, E.-S. Cho, J.-H. Park, and H.Y. Son, "Protective effect of Korean red ginseng against aflatoxin B1-induced hepatotoxicity in rat," Journal of ginseng research, vol. 35, no. 2, pp. 243-249, 2011.

[34] J. Gao, Z. Yu, S. Jing et al., "Protective effect of Anwulignan against D-galactose-induced hepatic injury through activating p38 MAPK-Nrf2-HO-1 pathway in mice," Clinical Interventions in Aging, vol. 13, pp. 1859-1869, 2018.

[35] O. Bayrak, N. Bavbek, Karatas OF et al., "Nigella sativa protects against ischaemia/reperfusion injury in rat kidneys," Nephrology Dialysis Transplantation, vol. 23, no. 7, pp. 22062212, 2008.

[36] F. Dong, S. Wang, Y. Wang et al., "Quercetin ameliorates learning and memory via the Nrf2-ARE signaling pathway in d-galactose-induced neurotoxicity in mice," Biochemical and Biophysical Research Communications, vol. 491, no. 3, pp. 636-641, 2017.

[37] D.-J. Kim, K.-S. Seong, D.-W. Kim, S.-R. Kim, and C.C. Chang, "Antioxidative effects of red ginseng saponins on paraquat-induced oxidative stress," Journal of Ginseng Research, vol. 28, no. 1, pp. 5-10, 2004.

[38] J. Y. Kim, J. Y. Park, H. J. Kang, O. Y. Kim, and J. H. Lee, “Beneficial effects of Korean red ginseng on lymphocyte DNA damage, antioxidant enzyme activity, and LDL oxidation in healthy participants: a randomized, double-blind, placebo-controlled trial," Nutrition Journal, vol. 11, no. 1, pp. 1-9, 2012.

[39] A. Valavanidis, T. Vlachogianni, and C. Fiotakis, "8-Hydroxy$2^{\prime}$-deoxyguanosine (8-OHdG): a critical biomarker of oxidative stress and carcinogenesis," Journal of environmental science and health Part C, vol. 27, no. 2, pp. 120-139, 2009.

[40] H. J. Choi, H. S. Han, J. H. Park et al., "Antioxidantive, phospholipase A2 inhibiting, and anticancer effect of polyphenol rich fractions from Panax ginseng CA Meyer," Journal of the korean Society of Agricultural and Biotechnology, vol. 46, no. 3, pp. 251-256, 2003.

[41] L.-Q. Xu, Y.-L. Xie, S.-H. Gui et al., "Polydatin attenuates dgalactose-induced liver and brain damage through its anti-oxidative, anti-inflammatory and anti-apoptotic effects in mice," Food \& Function, vol. 7, no. 11, pp. 4545-4555, 2016.

[42] I. E. Kochevar, "Phototoxicity mechanisms: chlorpromazine photosensitized damage to DNA and cell membranes," The Journal of Investigative Dermatology, vol. 77, no. 1, pp. 59$64,1981$. 